\title{
Dynamic Importance Sampling for the Escape Problem in Nonequilibrium Systems: Observation of Shifts in Optimal Paths
}

\author{
S. Beri, ${ }^{1}$ R. Mannella, ${ }^{2,1}$ and P.V. E. McClintock ${ }^{1}$ \\ ${ }^{1}$ Department of Physics, Lancaster University, Lancaster LA1 4YB, United Kingdom \\ ${ }^{2}$ Dipartimento di Fisica, Università di Pisa and INFM UdR Pisa, Via Buonarroti 2, 56100 Pisa, Italy
}

(Received 17 July 2003; published 14 January 2004)

\begin{abstract}
The activation problem is investigated in two-dimensional nonequilibrium systems. A numerical approach based on dynamic importance sampling (DIMS) is introduced. DIMS accelerates the simulations and allows the investigation to access noise intensities that were previously forbidden. The escape path is observed to be shifted compared to a heteroclinic trajectory calculated in the limit of zero-noise intensity. A theory to account for such shifts is presented and shown to agree with the simulations for a wide range of noise intensities.
\end{abstract}

DOI: 10.1103/PhysRevLett.92.020601

PACS numbers: 05.40.-a, 02.50.Ng, 02.70.Tt

Activation processes lie at the heart of a large class of physical phenomena in nonequilibrium systems, such as stochastic resonance [1], directed diffusion in stochastic ratchets [2], nucleation in electrochemical systems [3], the dynamics of vertical cavity surface emitting lasers (VCSELs) [4] and gas lasers [5], and the passage of currents of ions through open ionic channels [6] in biological membranes. The Kramers activation process lies within this class of problems [7]. Despite its importance, the problem of activation cannot be regarded as solved. On the theoretical side, important steps towards the solution have been taken in the regime of zero-noise intensities. They predict the escape to take place in a ballistic manner along a most probable escape path (MPEP) that can be obtained from an auxiliary Hamiltonian system [8-14]. The same picture still applies even for escape at short times (i.e., before quasiequilibrium has been established) [15]. More recently it has been established [16] that finite noise does more than just changing the prefactor of the exponential. It causes the system to follow a path that differs from the zero-noise theoretical MPEP.

The activation problem has also been extensively studied numerically, in particular, using Monte Carlo simulations. However, even for simple systems, the simulation time grows exponentially with decreasing noise intensity. Consequently, the really interesting range of noise intensities, i.e., small enough for valid comparisons to be made with theoretical predictions, has in practice been inaccessible to numerical experiments. Intensive efforts are therefore being made to find ways of speeding up the simulations [17-19]. In [17] the probability distribution for the system is built up iteratively. In this way, the shift of singularities in the probability distribution and oscillation of the probability at the boundary due to finite noise intensity have been observed. These features are consistent with the predicted shift of optimal paths due to the finiteness of the noise intensity. However, simulations able to reveal the predicted shift in the optimal path with increasing noise intensity are still lacking: to address this question we need numerical approaches which yield, not the probability distributions, but rather the actual paths followed by the dynamics of the system of interest. Simulation techniques based on dynamic importance sampling (DIMS) were proposed in [18] for one-dimensional dynamical systems to study dynamics in the limit of very small noise intensity. DIMS aims to accelerate Monte Carlo integrations by adding an appropriately chosen biasing field to the equations of motion, which remain stochastic in nature. Standard techniques are then used to relate Monte Carlo results in the presence of the bias to the statistical observables of the original system.

Extensions of DIMS to higher-dimensional systems (like those one commonly encounters when dealing with nonequilibrium processes) are not straightforward. An earlier biasing technique, introduced in [19] for both one- and two-dimensional systems, was found incapable of detecting either the shift of the escape path [16] or saddle point avoidance [20], both of which had been predicted theoretically. Moreover, in the presence of singularities, a bad choice of bias might hide important physical features of the system of interest. Note that other numerical approaches, like the umbrella sampling technique [21], are not appropriate for nonequilibrium systems.

In this Letter we propose a robust generalization of DIMS that is, in principle, valid for an arbitrary number of dimensions. The aim is to reveal finite noise features in the vicinity of the MPEP, accelerating the simulation by exploitation of well-established zero-noise-limit theoretical results. We apply this technique to the investigation of escape in multidimensional nonequilibrium systems for very small but finite noise intensities, thereby accessing a range far beyond anything investigated previously. As we show, the predicted shift of the optimal path due to the finiteness of the noise is clearly resolved and turns out to be in excellent agreement with theory [16] over more than 4 orders of magnitude in the noise intensity. 
We first describe the problem for a generic nonlinear system under the influence of noise

$$
\begin{gathered}
\dot{x}=f(x)+\xi(t), \\
\langle\xi(t)\rangle=0 \quad\langle\xi(t) \xi(s)\rangle=\epsilon \delta(t-s) .
\end{gathered}
$$

Here $x$ represents a vector of coordinates, $f(x)$ is a nonlinear function, and $\xi(t)$ is a family of zero-mean uncorrelated noise processes of intensity $\epsilon$. For systems in detailed balance [for example, if $f(x)$ is the gradient of some potential function], activation traverses paths that are time reversed relaxational trajectories [22]. In what follows we consider the case where $f(x)$ does not satisfy the detailed balance condition, and we focus on the important case of $f(x)$ showing unstable cycles coexisting with stable steady states. The stochastically equivalent Fokker-Planck equation for the probability density is

$$
\frac{\partial \varrho}{\partial t}=-\frac{\partial(f \varrho)}{\partial x}+\frac{\epsilon}{2} \frac{\partial^{2} \varrho}{\partial x^{2}} \text {. }
$$

In the limit $\epsilon \rightarrow 0$, (3) can be solved using the WKB expansion [8,9]. Inserting a test function of the form

$$
\varrho(x, t)=z(x, t) \exp \left(-\frac{S(x, t)}{\epsilon}\right), \quad \epsilon \rightarrow 0,
$$

in (3), at leading order $\epsilon^{-1}$, the auxiliary function $S$ turns out to be the solution of the Hamilton-Jacobi equation for a classical action

$$
\frac{\partial S}{\partial t}=H\left(x, \frac{\partial S}{\partial x}\right)
$$

with the Hamiltonian equations

$$
\dot{x}=f(x)+p \quad \dot{p}=-p \frac{d f}{d x} .
$$

The Hamiltonian for the system, $H(x, p)=\frac{p^{2}}{2}+p \cdot f$, is known as the Wentzell-Freidlin Hamiltonian [9]. The function $S$ evolves along the characteristics of (5) according to $\dot{S}=\frac{1}{2} p^{2}$. Being nondecreasing along trajectories and differentiable almost everywhere, the function $S$ can be considered as a nonequilibrium potential for the system [23-26]. It is known from the theory of dynamical systems that $S(x, t)$ can be a multivalued function of position in the coordinate space $[23,25,27,28]$. In this case, more than one trajectory solution of (5) reaches the same point in coordinate space. In the limit $\epsilon \rightarrow 0$, only the least action $S_{\min }$ is relevant, and all trajectories corresponding to higher values of $S$ are exponentially disadvantaged. Escape takes place along the least action trajectory with overwhelming probability in the limit $\epsilon \rightarrow 0$. This is the MPEP, and the action calculated along it is the "activation energy." Regions of coordinate space reached by different families of optimal paths are separated by switching lines (SL) [11,12,29]. A trajectory ceases to be optimal after crossing a switching line. For this reason, the most probable escape path can touch the SL only asymptotically. Because the SL reaches the cycle asymptotically [12], the only possible optimal escape trajectories are heteroclinic paths connecting the initial state to the saddle cycle in infinite time.

To calculate the effect of a finite small $\epsilon \neq 0$ in this picture, a calculation of the next-to-the-leading order in the WKB expansion is performed. In this order of approximation, the prefactor $z(x, t)$ satisfies

$$
\frac{d z}{d t}=-z \partial_{i} f^{i}-\frac{1}{2} z \partial_{i} \partial_{j} S
$$

[11], where the notation $\partial_{i}$ has been used for $\frac{\partial}{\partial x_{i}}$. The solution of (7) calls for a knowledge of the second derivatives of the action calculated along the characteristics. Differentiating twice (5), the following equations are obtained [11]:

$$
\begin{aligned}
\frac{d}{d t}\left(\frac{\partial^{2} S}{\partial x^{i} \partial x^{j}}\right)= & -\frac{\partial^{2} H}{\partial x^{i} \partial x^{j}}-\frac{\partial^{2} S}{\partial x^{i} \partial x^{k}} \frac{\partial^{2} H}{\partial p^{k} \partial x^{j}} \\
& -\frac{\partial^{2} S}{\partial x^{j} \partial x^{l}} \frac{\partial^{2} H}{\partial x^{i} \partial p^{l}}-\frac{\partial^{2} S}{\partial x^{j} \partial x^{l}} \frac{\partial^{2} S}{\partial x^{i} \partial x^{k}} \frac{\partial^{2} H}{\partial p^{k} \partial p^{l}} .
\end{aligned}
$$

At this order of approximation, the nonequilibrium potential has to be corrected as $S_{\epsilon}=S-\epsilon \log (z)$. The position of the switching line is now defined as $S_{\epsilon}^{1}=S_{\epsilon}^{2}$, where the indices 1,2 refer to two different trajectories reaching the same point in the coordinate space [17]. The modified switching line does not touch the cycle asymptotically but hits it at a definite position. As a consequence, heteroclinic trajectories are no longer optimal and optimal escape follows a path different than MPEP.

To test these ideas, we propose an extension of DIMS $[18,19]$ to the multidimensional case. It is suitable for unraveling features of the topology close to the optimal escape path in the limit of small but finite noise intensity. The method is applicable to a system characterized by a stable attractor with a sizable basin of attraction, where escape takes place via a large fluctuation.

For a given dynamical system, we initially set the system close to the stable attractor and integrate the exact equations of motion using a standard SDE (stochastic differential equation) integrator: for the examples shown below, we used the Heun integrator [30]. We also set a boundary at some distance from the stable attractor: this boundary has nothing to do with the boundary of the basin of attraction, but it defines when we switch from standard SDE integration to DIMS integration. Although the final result does not appear to depend on the boundary choice, nevertheless the boundary ought to be chosen on some rational basis: we require that the boundary should be fairly easily reached by the dynamical system when using straight SDE integration, but also that this event should not be too probable. In practice, a reasonable boundary could be, e.g., a circle centered on the stable attractor, with a radius of a few $\sqrt{\epsilon}$. This choice is reproducing the diffusive dynamics in the vicinity of 
the stable state. When the system hits the boundary, we switch to the DIMS integrator: from the above discussion, we know that the SDE

$$
\dot{x}=f(x)+\xi(t)
$$

maps onto the Hamiltonian equation (6), so we switch to the integration of

$$
\dot{x}=f(x)+p(x)+\xi(t),
$$

where $p(x)$ is the momentum conjugated with $x$ as obtained from the MPEP and $\xi(t)$ is the DIMS stochastic component. It is clear that in more than one dimension the probability of being exactly on the MPEP during each integration time step is negligible, but the idea is, nevertheless, that we should use in the integration of (9) the value of $p(x)$ which "pulls" the escape tube towards the boundary. This is achieved by linearizing the Hamilton equations near the MPEP and using the $p(x)$ that keeps the system on the stable manifold of the MPEP. A step of the scheme is explained in Fig. 1. Consider point $A$ in the coordinate space. The momentum $p(x)$ to be used in Eq. (9) is calculated in the following way: point $A^{*}$ in the extended phase space which has the same $x$ coordinate as $A$, and lies on the stable manifold of the MPEP, is located. The momentum of point $A^{*}$ is then used as $p(x)$ in (9). The integration scheme is interrupted and reset if the trajectory leaves a small neighborhood of the MPEP (size of a few $\sqrt{\epsilon}$ ).

We now consider two explicit examples of the application of this algorithm. First, we consider the inverted Van der Pol oscillator (IVDP). It is characterized by the presence of an unstable cycle with a stable point at its center. DIMS is used here to study transitions from the stable state to the limit cycle. The IVDP system is described by the following set of equations:

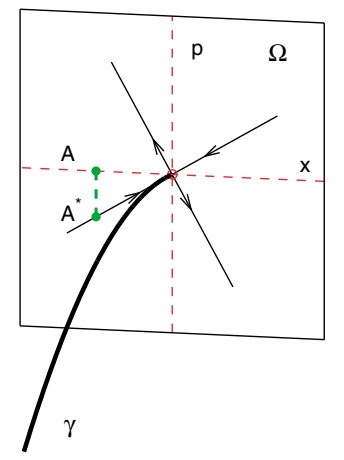

FIG. 1 (color online). The scheme for one step of the DIMS method, where $\Omega$ is a cross section in the extended phase space, $\gamma$ (thick line) is the optimal path predicted by the theory, and the thin lines are the stable and unstable eigenspaces (as indicated by the directions of the arrows) of the optimal path. The dashed lines represent, respectively, the coordinate space $x$ and the momentum space $p . A$ is a point in the coordinate space; $A^{*}$ is the corresponding point on the stable manifold of $\gamma$ as required by the integration procedure.

$$
\dot{x}=y, \quad \dot{y}=-x-2 \eta\left(1-x^{2}\right) y+\sqrt{\epsilon} \xi(t) .
$$

Here $x$ and $y$ are dynamical variables and $\xi(t)$ is a white Gaussian process of unit intensity. The DIMS simulation have been performed for a range of intensities running between $10^{-5}$ and $10^{-2}$. The results are shown in Fig. 2 for two different noise intensities. The agreement between the theoretical and experimental escape paths at finite noise intensities is seen to be excellent. The shift in the optimal path is dramatic, leading to a marked change in the point where it hits the cycle.

As a second example, we consider the harmonically driven overdamped Duffing oscillator

$$
\dot{x}=x-x^{3}+A \sin (\omega t)+\sqrt{\epsilon} \xi(t) .
$$

Here $x$ is the dynamical coordinate, $A$ and $\omega$ are the amplitude and the frequency of the external driving, and $\xi$ is a white Gaussian process of unit intensity. The parameters $A$ and $\omega$ are chosen to be in the nonperturbative, nonadiabatic regime. The dynamics of this system is characterized by two stable limit cycles separated by an unstable limit cycle. In the presence of noise, the system can fluctuate from one stable cycle to the other. Some simulation results are shown in Fig. 3. The experimental trajectory follows closely the heteroclinic path when it is far from the stable and unstable cycles. As the system approaches the unstable cycle, however, diffusion becomes important and the escape then runs along the finite noise escape path calculated using finite noise corrections. Experiments with different noise intensities (not shown) reveal an increase of the shift with noise intensity.

In conclusion, we have introduced a highly efficient way of performing numerical simulations, based on the idea of taking the momentum so as to stay on the stable manifold. It is applicable to a large class of dynamical systems. The technique provides a unique tool for studying features induced by finite noise in the neighborhood of
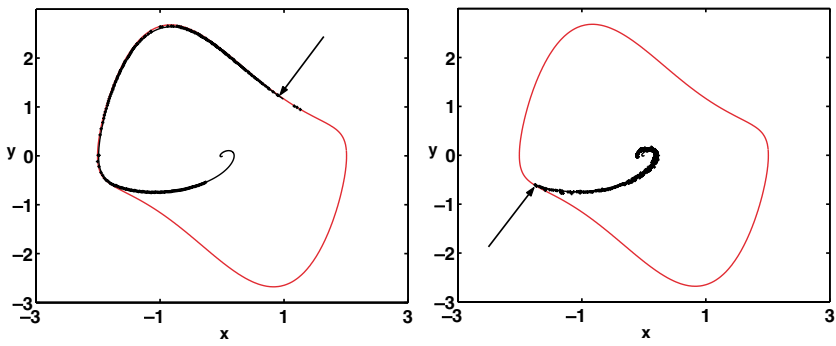

FIG. 2 (color online). Simulations of escape in the IVDP system (9), with $\eta=0.5$ and two different noise intensities: $\epsilon=10^{-5}$ (left) and $\epsilon=10^{-2}$ (right). The full lines are the limit cycle and the most probable escape path; the crosses are points on the ridge of the prehistory probability density for the escape. The arrows indicate the positions where the theoretical modified optimal escape path hits the cycle. For both noise intensities, the theory agrees well with the experimental points. A significant shift of the optimal path is evident. 


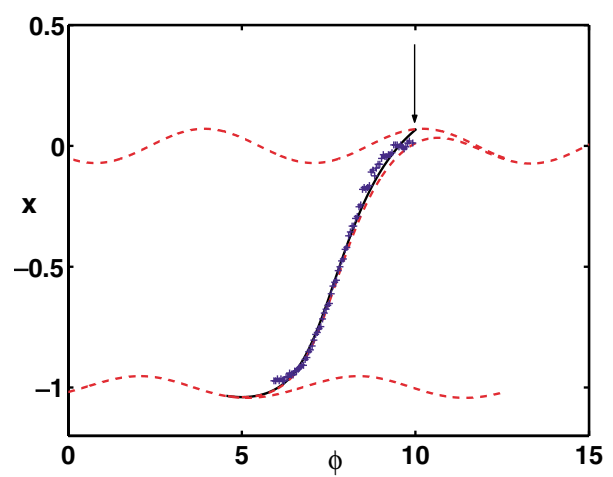

FIG. 3 (color online). Simulations of escape in the Duffing system (10), with $A=0.1, \omega=1$, and $\epsilon=4 \times 10^{-3}$. The steady states and the most probable escape path at $\epsilon=0$ are indicated by dashed lines. The noise corrected optimal path is shown by a solid line. The crosses are points on the ridge of the prehistory probability density for the escape. The arrow shows where the noise-corrected optimal path hits the cycle.

the optimal escape path and hence leads to a biasing scheme that is based on the dynamics of the system itself, thus constituting a most natural scheme for dynamic importance sampling. Using this method we were able to probe new regimes of low but finite noise intensity and were able to observe experimentally the shift of escape path predicted in [17]; we stress that this shift is a large physical effect, even at the smallest noise intensities investigated in this Letter. The technique provides a way of extending experimental studies to noise intensities that were previously inaccessible and promises to accelerate research in many fields of science, e.g., in the investigation of the polarization of flip dynamics in VCSELs or in the motion of ions in the pores of cellular membranes. It will be straightforward to extend the method to treat the case of a stochastic nonlinear map like the one used in [5].

We are grateful for valuable discussions with Dmitri Luchinsky and Stanislav Soskin. The research has been supported in part by the Engineering and Physical Sciences Research Council (U.K.) and by INTAS.

[1] M. I. Dykman, D. G. Luchinsky, R. Mannella, P.V. E. McClintock, N. D. Stein, and N. G. Stocks, Nuovo Cimento Soc. Ital. Fis. 17D, 661 (1995); L. Gammaitoni, P. Hänggi, and F. Marchesoni, Rev. Mod. Phys. 70, 223 (1998).

[2] F. Jülicher, F. Ajdari, and J. Prost, Rev. Mod. Phys. 69, 1269 (1997); M. O. Magnasco, Phys. Rev. Lett. 71, 1477 (1993); M. I. Dykman, H. Rabitz, V. N. Smelyanskiy, and B. E. Vugmeister, Phys. Rev. Lett. 79, 1178 (1997).

[3] V. N. Smelyanskiy, M. I. Dykman, H. Rabitz, B. E. Vugmeister, S. L. Bernasek, and A. B. Bocarsly, J. Chem. Phys. 110, 11488 (1999).
[4] M. B. Willemsen, M. P. van Exter, and J. P. Woerdman, Phys. Rev. Lett. 84, 4337 (2000); M. B. Willemsen, M. P. van Exter, and J. P. Woerdman, Phys. Rev. A 604105 (1999); M. P. van Exter, R. F. M. Hendriks, and J.P. Woerdman, Phys. Rev. A 57, 2080 (1998); M. P. van Exter, M. B. Willemsen, and J. P. Woerdman, Phys. Rev. A 58, 4191 (1998).

[5] V. A. Gaisyonok, E. V. Grigorieva, and S. A. Kashchenko, Opt. Commun. 124, 408 (1996); V. N. Chizhevsky, E.V. Grigorieva, and S. A. Kashchenko, Opt. Commun. 133, 189 (1997).

[6] R. S. Eisenberg, M. M. Klosek, and Z. Schuss, J. Chem. Phys. 102, 1767 (1995).

[7] H. A. Kramers, Physica (Utrecht) 7, 284 (1940); M. I. Dykman, Phys. Rev. A 42, 2020 (1990); V. I. Mel'nikov, Phys. Rep. 209, 1 (1991); P. Hänggi, P. Talkner, and M. Borkovec, Rev. Mod. Phys. 62, 251 (1990).

[8] D. Ludwig, SIAM Rev. 17, 605 (1975).

[9] M. Freidlin and A. D. Wentzel, Random Perturbations in Dynamical Systems (Springer, New York, 1984).

[10] R. S. Maier and D. L. Stein, Phys. Rev. Lett. 71, 1783 (1993); R. S. Maier and D. L. Stein, Phys. Rev. Lett. 85, 1358 (2000).

[11] R. S. Maier and D. L. Stein, J. Stat. Phys. 83, 291 (1996).

[12] V. N. Smelyanskiy and M. I. Dykman, Phys. Rev. E 55, 2516 (1997).

[13] M. I. Dykman, M. M. Millonas, and V. N. Smelyanskiy, Phys. Lett. A 195, 53 (1994).

[14] J. Lehmann, P. Reimann, and P. Hänggi, Phys. Rev. E 62, 6282 (2000)

[15] S. M. Soskin, V. I. Sheka, T. L. Linnik, and R. Mannella, Phys. Rev. Lett. 86, 1665 (2001).

[16] A. Bandrivskyy, S. Beri, and D. G. Luchinsky, Phys. Lett. A 314, 386 (2003).

[17] A. Bandrivskyy, S. Beri, D. G. Luchinsky, R. Mannella, and P.V.E. McClintock, Phys. Rev. Lett. 90, 210201 (2003).

[18] D. M. Zuckermann and T. B. Woolf, Phys. Rev. E 63, 016702 (2001).

[19] T. B. Woolf, Chem. Phys. Lett. 289, 433 (1998).

[20] D. G. Luchinsky, R. S. Maier, R. Mannella, P.V.E. McClintock, and D. L. Stein, Phys. Rev. Lett. 82, 1806 (1999).

[21] C. Dellago, P. G. Bolhouis, F. S. Csajka, and D. Chandler, J. Chem. Phys. 108, 1964 (1998).

[22] D. G. Luchinsky and P.V.E. McClintock, Nature (London) 389, 463 (1997).

[23] R. Graham and T. Tel, Phys. Rev. Lett. 52, 9 (1984).

[24] R. Graham and T. Tel, Phys. Rev. A 31, 1109 (1985).

[25] H. R. Jauslin, Physica (Amsterdam) 144A, 179 (1987).

[26] R. Graham, in Noise in Nonlinear Dynamical Systems, edited by F. Moss and P.V.E. McClintock (Cambridge University Press, Cambridge, 1989), Vol. 1, pp. 225-278.

[27] V. Arnold, Mathematical Methods of Classical Mechanics (Springer-Verlag, Berlin, 1978).

[28] M. V. Day, Stochastics 20, 121 (1987).

[29] M. I. Dykman et al., Phys. Rev. Lett. 68, 2718 (1992).

[30] The Heun algorithm has been extensively described and tested in the literature; see, for instance, R. Mannella, Int. J. Mod. Phys. C 13, 1177 (2002), and references therein. 